SSCL

SFU-Preprint-92-5

\title{
Comments on the Vacuum Orientations in QCD
}

\author{
Zheng Huang and K.S. Viswanathan \\ Department of Physics, Simon Fraser University \\ Burnaby, B.C. V5A 1S6, Canada \\ Dan-di $\mathbf{W u}^{*}$ \\ Superconducting Super Collider Laboratory ${ }^{\dagger}$ \\ 2550 Beckleymeade Avenue, Dallas, Texas 75237
}

\begin{abstract}
We study the QCD vacuum orientation angles in correlation with the strong CP phases. A vacuum alignment equation of the dynamical chiral symmetry breaking is derived based on the anomalous Ward identity. It is emphasized that a chiral rotation of the quark field causes a change of the vacuum orientation and a change in the definition of the light pseudoscalar generators. As an illustration of the idea, $\eta \rightarrow 2 \pi$ decays are carefully studied in different chiral frames. Contrary to the claim in Ref.[7], the $\theta$-term does not directly contribute to the CP-violating processes.
\end{abstract}

May 12, 2019

* On leave of absence from School of Physics, University of Melbourne, Parkville, Vic 3052, Australia.

$\dagger$ Operated by the University Research Association, In., for the U.S. Department of Energy under Contract No. DE-AC35-89ER40486. 
In QCD lagrangian of strong interactions, there are two possible sources of $\mathrm{CP}$ violation: the complex quark mass terms and the $\theta$-term. It has been long realized that they are related to each other by chiral transformations associated with the quark fields. The physical effects of $\mathrm{CP}$ violation only depend on a chiral-rotation invariant $\bar{\theta}$ defined as

$$
\bar{\theta}=\theta_{Q C D}+\theta_{Q F D}=\theta_{Q C D}+\sum_{i}^{L_{f}} \phi_{i}
$$

where $\theta_{Q C D}$ is the coefficient of the $\theta$-term, $\phi_{i}$ is the phase of the ith quark mass term, and $L_{f}$ is the number of light quarks]. However, there is another source of CP-violating angles, the phases of the quark condensates, which arise from dynamical chiral symmetry breaking (DCSB)

$$
\left\langle\bar{\psi}_{L}^{i} \psi_{R}^{i}\right\rangle=-\frac{C_{i}}{2} e^{i \beta_{i}} ;\left\langle\bar{\psi}_{R}^{i} \psi_{L}^{i}\right\rangle=-\frac{C_{i}}{2} e^{-i \beta_{i}}
$$

where $\psi$ is the quark field and $C_{i}$ 's, $\beta_{i}$ 's are real. The $\mathrm{QCD}$ vacuum orientation is characterized by a set of phases of the quark condensates. If the vacuum angle $\beta_{i} \neq 0$ it follows that $\left\langle\bar{\psi}^{i} i \gamma_{5} \psi^{i}\right\rangle=C_{i} \sin \beta_{i} \neq 0$ which may also break CP symmetry since $\left\langle\bar{\psi} i \gamma_{5} \psi\right\rangle$ is a P-odd and CP-odd quantity. It has been proven by Vafa and Witten that when $\bar{\theta}=0$ and $\phi_{i}=0$ for all $i$ 's, the parity symmetry in a vector-like theory such as QCD is not spontaneously broken [四] therefore $\beta_{i}=0$ or $\pi$ [2] for all $i$ 's. When $\bar{\theta} \neq 0$, on the other hand, one generally expects that the $\mathrm{CP}$-violating interactions in the lagrangian may result in a $\mathrm{CP}$-asymmetric physical vacuum. The purpose of the present paper is to study the vacuum orientation in the presence of strong $\mathrm{CP}$ violation and its potential effects on $\mathrm{CP}$-violating processes in strong interactions. We find that the phases of quark condensates can be completely determined as functions of $\bar{\theta}$ and $\phi_{i}$ 's via a vacuum alignment equation. Thus $\beta_{i}$ 's are not spontaneously generated either even when the CP symmetry is explicitly violated by $\bar{\theta} \neq 0$.

Obviously, the quark condensates (2) cannot be referred to as fundamental parameters of the theory since they are subject to chiral transformations. In fact $\beta_{i}$ 's can be set to any values if we make appropriate chiral transformations for the quark fields. Such transformations

${ }^{1}$ The inclusion of heavy quarks will not change our discussion significantly if they are in the normal phase. Otherwise see Ref. [4]. 
also change the phases of the quark masses, as well as the coefficient of the $\theta$-term because of the chiral anomaly. But what is important is the correlation between the vacuum orientation and the distribution of the strong CP phases among $\theta$-term and quark mass terms, which is to be determined by the vacuum alignment. The effective CP-violating interactions in low energy hadron physics (for instance, in current algebra) highly depend on this correlating feature. As we shall see below, the sole $\bar{\theta}$-dependence of the strong CP effects is proven only when the orientations of the vacuum are properly considered. In addition, it is of interest to study DCSB in the presence of strong CP violation in its own right.

One way of relating the phases of the quark condensates with $\theta_{Q C D}$ and $\phi_{i}$ 's is to consider the so-called anomalous Ward identity [3]

$$
\begin{aligned}
\frac{1}{2} \partial^{\mu} J_{\mu}^{(i) 5}= & F \tilde{F}+i m_{i} e^{i \phi_{i}} \bar{\psi}_{L}^{i} \psi_{R}^{i}-i m_{i} e^{-i \phi_{i}} \bar{\psi}_{R}^{i} \psi_{L}^{i} \\
& \left(i=1,2, \ldots, L_{f}\right)
\end{aligned}
$$

where $J_{\mu}^{(i) 5}=\bar{\psi}^{i} \gamma_{\mu} \gamma_{5} \psi^{i}, F \tilde{F}=\frac{g^{2}}{32 \pi^{2}} \epsilon_{\mu \nu \rho \sigma} F^{\mu \nu} F^{\rho \sigma}$ and $F^{\mu \nu}$ the non-abelian gauge field strength tensor. Taking the vacuum expectation values (VEV) on both sides of (3) yields [4]

$$
\begin{aligned}
\langle F \tilde{F}\rangle & =-i m_{i}\left[e^{i \phi_{i}}\left\langle\bar{\psi}_{L}^{i} \psi_{R}^{i}\right\rangle-e^{-i \phi_{i}}\left\langle\bar{\psi}_{R}^{i} \psi_{L}^{i}\right\rangle\right] \\
& =-m_{i} C_{i} \sin \left(\phi_{i}+\beta_{i}\right) \quad\left(i=1,2, \cdots, L_{f}\right) .
\end{aligned}
$$

In deriving (4a) we have assumed that the VEV's of the divergence of the gauge invariant current vanish. Eq.(4b) is the master equation of this paper. It is important to point out that if the DCSB does not occur, (4a) would vanish identically and there is no constraint on those phases. Indeed even though the quark condensate can be non-zero due to the explicit chiral symmetry breaking (ECSB) i. e. the quark current masses, it does not contribute to (4a) because it possesses a phase opposite to the phase of the quark mass $\phi_{i}$ and renders the RHS of (4a) zero. This can be easily seen by taking the free-quark limit in which the condensate is calculated as

$$
\left\langle\bar{\psi}_{L}^{i} \psi_{R}^{i}\right\rangle=\frac{1}{2} \int \frac{d^{4} k}{(2 \pi)^{4}} \operatorname{Tr} \frac{1+\gamma_{5}}{\not k-m_{i} e^{i \phi_{i} \gamma_{5}}}=-m_{i} \Lambda^{2}\left(m_{i}\right) e^{-i \phi_{i}}
$$


where $\Lambda^{2}$ is real. The substitution of (5) into (4a) yields $\langle F \tilde{F}\rangle=0$. Therefore, $C_{i}$ 's in (4a) should be understood as the purely dynamical condensates originating from DCSB. The kinematical part of the condensates that is induced by the ECSB and has a phase $-\phi_{i}$ has been subtracted out in (4a). It is the DSCB combining with the topological structure of QCD Yang-Mills fields (the instanton effect) that makes the strong CP phases non-trivial and relates them to each other.

Eq. (4b) is not immediately useful to us since it has an unknown quantity $\langle F \tilde{F}\rangle$. It involves no quark fields thus is independent of the chiral transformation. It is conceivable that $\langle F \tilde{F}\rangle$ is solely a function of $\bar{\theta}$ (not of $\phi_{i}$ 's and $\beta_{i}$ 's separately). A rigorous proof can be made by summing over instanton configurations in QCD $\theta$ vacua. For simplicity, consider QCD with a single quark field $\psi$. The VEV's of $F \tilde{F}$ is given by [5]

$$
\begin{aligned}
\langle F \tilde{F}\rangle & =\frac{1}{V T}\left\langle\int d^{4} x F \tilde{F}\right\rangle \\
& =\frac{1}{V T} \frac{1}{N} \sum_{\nu=0, \pm 1, \cdots} e^{i \bar{\theta} \nu} \nu \int\left[d A_{\mu}\right]_{\nu} \operatorname{det}\left(i \not D_{\nu}+i m\right) \exp \left(-\int d^{4} x F F\right)
\end{aligned}
$$

where $N$ is the normalization factor, $V T$ is the volume of Euclidean space-time, and $\nu$ is the winding number of the instanton field configuration, and the fermion determinant results from the integration over the quark field. We have made an appropriate chiral transformation such that the quark mass is real and $\theta_{Q C D}=\bar{\theta}$ (we can always do so because the generating functional is invariant under the redefinition of integral variables). It is shown that when $\nu>0(<0) i \not D_{\nu}$ has $|\nu|$ zero modes with negative (positive) chirality [6]. We thus obtain

$$
\begin{aligned}
\langle F \tilde{F}\rangle & =m A_{1}\left(m^{2}\right) \frac{-i}{2}\left(e^{i \bar{\theta}}-e^{-i \bar{\theta}}\right) \\
& +m^{2} A_{2}\left(m^{2}\right) \frac{-i}{2}\left(e^{i 2 \bar{\theta}}-e^{-i 2 \bar{\theta}}\right)+\cdots+m^{|\nu|} A_{|\nu|}\left(m^{2}\right) \frac{-i}{2}\left(e^{i|\nu| \bar{\theta}}-e^{-i|\nu| \bar{\theta}}\right)+\cdots \\
& =m A_{1}\left(m^{2}\right) \sin \bar{\theta}+m^{2} A_{2}\left(m^{2}\right) \sin 2 \bar{\theta}+\cdots=K(m, \theta) \sin \bar{\theta}
\end{aligned}
$$

where $A_{|\nu|}\left(m^{2}\right)$ 's are given in Euclidean space

$$
A_{|\nu|}\left(m^{2}\right)=\frac{1}{V T N} e^{-|\nu| \frac{8 \pi^{2}}{g^{2}}} \int\left[d A_{\mu}\right]_{\nu} \prod_{\lambda_{r}>0}\left[\lambda_{r}^{2}(A)+m^{2}\right] \exp \left[-\int d^{4} x F F\right] .
$$


Here $\lambda_{r}(A)$ 's are non-zero eigenvalues of $i \not D_{\nu}$. Clearly $A_{|\nu|}\left(m^{2}\right)$ 's are some real functions of $m^{2}$ and do not vanish as $m \rightarrow 0$. If $\bar{\theta}$ is small as it must be, $\langle F \tilde{F}\rangle \simeq K(m) \bar{\theta}=$ $K(m) \theta_{Q C D}+K(m) \theta_{Q F D}$

Combining (7) with (4b), we derive the so-called vacuum alignment equation (VAE) [4, \&], which determines the orientation of the QCD vacuum in the presence of strong $\mathrm{CP}$ violation

$$
\begin{gathered}
K(m) \bar{\theta}=m_{i} C_{i}\left(\phi_{i}+\beta_{i}\right)+O\left(m^{2} ; \bar{\theta}^{2}\right) \\
\left(i=1,2, \cdots, L_{f}\right) .
\end{gathered}
$$

Eq.(9) has proven that $\beta_{i}$ 's are not spontaneously generated even when $\bar{\theta} \neq 0$. The conclusion of Vafa and Witten's theorem [1] can be extended to the case where parity symmetry is explicitly violated. A similar result has been worked out previously [8] from different points of view. If $m_{i}$ 's vanish, $\beta_{i}$ 's can be arbitrary. This is referred to as the degeneracy of QCD vacua when the ECSB is absent. Any vacuum characterized by a set of the vacuum angles $\beta_{i}$ 's is as good as others and the orientation of the DCSB is arbitrary. However, the importance of (9) is that when the ECSB is turned on, the ground state must align with it in such a way that (9) is satisfied. Though both $\phi_{i}$ and $\beta_{i}$ are not physical parameters and can be changed through chiral rotations, their sum is uniquely determined by the physical parameter $\bar{\theta}$. When one is chosen the other is completely determined through making the vacuum alignment. As is emphasized by Dashen [9], a misaligned vacuum, whose orientation angles do not satisfy (9), may cause an inconsistency such as the goldstone bosons (pions) acquiring negative mass squares.

Once the DCSB and the ECSB align with each other, an absolute rotation of the whole system is of no concern. Thus a chiral transformation is allowed only if the corresponding change of the vacuum orientation has been taken into account. We can have two ways to make the vacuum alignment. We may choose one particular vacuum, for example, by requiring the quark condensate to be real $\beta_{i}=0\left(i=1,2, \cdots, L_{f}\right)$ and ask what perturbation (the ECSB) is aligned with it. Recalling that $C_{i}$ 's are dynamical condensates and thus $C_{i}=C_{j}=C$ 
$\left(i, j=1,2, \cdots, L_{f}\right)$, we obtain by solving $(9)$ for $\phi_{i}$ 's, to $O(m ; \bar{\theta})$

$$
\begin{gathered}
(A) \quad \phi_{i}=\frac{K(m)}{m_{i}} \bar{\theta} ; \quad \beta_{i}=0 \quad\left(i=1,2, \cdots, L_{f}\right) \\
\theta_{Q F D}=\sum_{i} \phi_{i}=\frac{K(m)}{\bar{m}} \bar{\theta} \quad ; \quad \theta_{Q C D}=\bar{\theta}-\theta_{Q F D}=\frac{K(m)-\bar{m}}{\bar{m}} \bar{\theta}
\end{gathered}
$$

where $\bar{m}=\left(\sum_{i} \frac{1}{m_{i}}\right)^{-1}$ and the CP-violating lagrangian

$$
\mathcal{L}_{(A)}^{C P}=-\sum_{i} m_{i} \phi_{i} \bar{\psi}^{i} i \gamma_{5} \psi^{i}+\theta_{Q C D} F \tilde{F}=-\frac{K(m)}{\bar{m}} \bar{\theta} \bar{\psi} i \gamma_{5} I \psi+\frac{K(m)-\bar{m}}{\bar{m}} \bar{\theta} F \tilde{F}
$$

where $I$ is an identity matrix. We shall call the solution (10) basis (A). Another way is to assume a certain pattern of the ECSB and to ask which one of the degenerate vacua corresponds to the perturbation. For example, we may choose the quark mass terms real $\phi_{i}=0\left(i=1,2, \cdots, L_{f}\right)$ and determine the vacuum angle $\beta_{i}$ 's. Again, from (9) we have

$$
\begin{aligned}
& \text { (B) } \quad \phi_{i}=0 \quad ; \quad \beta_{i}=\frac{K(m)}{m_{i}} \bar{\theta} \quad\left(i=1,2, \cdots, L_{f}\right) \\
& \theta_{Q F D}=\sum_{i} \phi_{i}=0 \quad ; \quad \theta_{Q C D}=\bar{\theta}
\end{aligned}
$$

and

$$
\mathcal{L}_{(B)}^{C P}=\bar{\theta} F \tilde{F}
$$

Solution (12) is to be called basis (B). We would like to emphasize again that by performing the chiral rotation on quark fields one has switched the strong CP phases among the $\theta$-term and quark mass terms, and obtained different lagrangians, each of which corresponds to a certain vacuum orientation. When calculating the strong CP effects we must take this into consideration to assure the correct result.

As an illustration, we compute the CP-violating $\eta \rightarrow 2 \pi$ decays in two bases with a given $\bar{\theta}$. In basis (A) where the condensates are real, we apply the soft-pion theorem to extracting $\eta$ and $\pi$ 'st

$$
\mathcal{A}\left(\eta \rightarrow \pi^{+} \pi^{-}\right)=\left\langle\pi^{+} \pi^{-}\left|\mathcal{L}_{(A)}^{C P}\right| \eta\right\rangle
$$

\footnotetext{
${ }^{2}$ We have worked in the context of $S U(3)_{L} \times S U(3)_{R}$ where $\eta$ and $\pi$ 's are all light pseudoscalars.
} 


$$
\begin{aligned}
& =-\frac{K(m)}{\bar{m}} \bar{\theta}\left(\frac{-i}{F_{\pi}}\right)^{3}\left\langle\left[ Q_{5}^{8},\left[Q_{5}^{+},\left[Q_{5}^{-}, \bar{\psi} i \gamma_{5} I \psi\right]\right\rangle\right.\right. \\
& \simeq-\frac{K(m)}{\bar{m}} \bar{\theta} \frac{1}{F_{\pi}}\left\langle\overline { \psi } \left\{\frac{\lambda^{8}}{2},\left\{\frac{\lambda^{+}}{2},\left\{\frac{\lambda^{-}}{2}, I\right\} \psi\right\rangle=\frac{K(m)}{\bar{m}} \bar{\theta} \frac{1}{F_{\pi}^{3}} \frac{2}{\sqrt{3}} C\right.\right.
\end{aligned}
$$

where the pion decay constant $F_{\pi} \approx 93 M e V$ and we have used $\left[Q_{5}^{a}, F \tilde{F}\right]=0$. The broken generators of $S U(3)_{A}$ corresponding to light pseudoscalars are given by

$$
Q_{5}^{a}=\int d^{3} x \psi^{\dagger} \gamma_{5} \frac{\lambda^{a}}{2} \psi(x) \quad(a=1,2, \cdots, 8)
$$

where $\lambda^{a}$ 's are Gell-Mann matrices and $\lambda^{ \pm}=1 / \sqrt{2}\left(\lambda_{1} \mp i \lambda_{2}\right)$. (14) has been first derived by Crewther, Di Vicchia, Veneziano and Witten (CDVW) [10]. However, there have been doubts about the calculation since it does not explicitly exhibit the use of the topological non-triviality of the $\theta$-vacuum. More concretely, one may shift the strong $\mathrm{CP}$ phases from $\theta_{Q F D}$ to $\theta_{Q C D}$ through chiral rotations and computes the amplitude, as one does in (14),

$$
\left\langle\pi^{+} \pi^{-}\left|\mathcal{L}_{(B)}^{C P}\right| \eta\right\rangle=\bar{\theta}\left(\frac{-i}{F_{\pi}}\right)\left\langle\pi^{-}\left|\left[Q_{5}^{-}, F \tilde{F}\right]\right| \eta\right\rangle
$$

which is zero if one imposes the canonical commutation relation by which $Q_{5}^{a}$ commutes with gauge fields. This contradiction has triggered a serious doubt on whether or not the strong CP phases lead to any physical effects at all [11.

We believe that this concern is redundant. The vacuum alignment equation (VAE) has incorporated the non-perturbative features of QCD vacuum into the game. Both $\mathcal{L}_{(A)}^{C P}$ and $\mathcal{L}_{(B)}^{C P}$ are solutions of the VAE and should, if one does things correctly, result in the same conclusion. In basis (B) the quark masses are real but the condensates are complex. The vacuum does not respect CP symmetry. In this case even though $\mathcal{L}_{(B)}^{C P}$ does not contribute to the amplitude as shown in (16), the CP conserving part of the lagrangian may do. Moreover, when the quarks have non-degenerate masses (mass splitting), the condensates are of the form

$$
\left\langle\bar{\psi}_{L} \psi_{R}\right\rangle=-\frac{C}{2} \beta \equiv-\frac{C}{2}(I+i \delta)
$$

with

$$
\beta=\left(\begin{array}{ccc}
e^{i \beta_{u}} & & \\
& e^{i \beta_{d}} & \\
& & \ddots
\end{array}\right) ; \delta \simeq\left(\begin{array}{lll}
\beta_{u} & & \\
& \beta_{d} & \\
& & \ddots
\end{array}\right)
$$


if $\beta_{i}$ 's are small. Apparently (17a) is not invariant under $S U\left(L_{f}\right)_{V}$ transformations if $\beta_{i}$ 's are not all equal. In other words, the vector charges defined as generators of $S U\left(L_{f}\right)_{V}$ do not annihilate the vacuum completely or

$$
Q^{a}|0\rangle \neq 0
$$

Clearly, the subgroup of $S U\left(L_{f}\right)_{L} \times S U\left(L_{f}\right)_{R}$ which leaves the vacuum invariant must satisfy

$$
U_{L}^{+} \beta U_{R}=\beta \quad \text { or } \quad U_{R}=\beta^{-1} U_{L} \beta
$$

where $U_{L}$ and $U_{R}$ are left and right unitary representations of $S U\left(L_{f}\right)$. The broken generators, which excite the glodstone bosons known as pions, are those of the coset of the unbroken group. From (19) it is easy to understand that the broken group is not $S U\left(L_{f}\right)_{A}$ any more but to be rotated to $\beta^{-1} S U\left(L_{f}\right)_{A} \beta$ generated by $\delta$. The pion generators, denoted by $\tilde{Q}_{5}^{a}$, are thus

$$
\tilde{Q}_{5}^{a}=\int d^{3} x\left\{\psi^{\dagger} \gamma_{5} \frac{\lambda^{a}}{2} \psi(x)+\psi^{\dagger}\left[\frac{\lambda^{a}}{2}, i \delta\right] \psi(x)\right\}+O\left(\delta^{2}\right),
$$

i. e. , the pions are mixing of P-odd and P-even components.

Now that $\mathcal{L}_{(B)}^{C P}=\bar{\theta} F \tilde{F}$ has no contribution to the amplitude, we have

$$
\begin{aligned}
\mathcal{A}\left(\eta \rightarrow \pi^{+} \pi^{-}\right)= & \left\langle\pi^{+} \pi^{-}|-\bar{\psi} m \psi| \eta\right\rangle \\
= & -\left(\frac{-i}{F_{\pi}}\right)^{3}\left\langle\left[\tilde{Q}_{5}^{8},\left[\tilde{Q}_{5}^{+},\left[\tilde{Q}_{5}^{-}, \bar{\psi} m \psi\right]\right\rangle\right.\right. \\
= & -\frac{i}{F_{\pi}^{3}}\left\{\left\langle\bar{\psi}\left[\left[\frac{\lambda^{8}}{2}, i \delta\right],\left\{\frac{\lambda^{+}}{2},\left\{\frac{\lambda^{-}}{2}, m\right\}\right\}\right] \psi\right\rangle+\left\langle\bar{\psi}\left\{\frac{\lambda^{8}}{2},\left[\left[\frac{\lambda^{+}}{2} i \delta\right],\left\{\frac{\lambda^{-}}{2}, m\right\}\right]\right\} \psi\right\rangle\right. \\
& \left.+\left\langle\bar{\psi}\left\{\frac{\lambda^{8}}{2},\left\{\frac{\lambda^{+}}{2},\left[\left[\frac{\lambda^{-}}{2}, i \delta\right], m\right]\right\}\right\} \psi\right\rangle-\left\langle\bar{\psi} \gamma_{5}\left\{\frac{\lambda^{8}}{2},\left\{\frac{\lambda^{+}}{2},\left\{\frac{\lambda^{-}}{2}, m\right\}\right\}\right\} \psi\right\rangle\right\}
\end{aligned}
$$

where $m$ is the diagonal mass matrix which is real in this basis. The first three terms in parenthesis come from the modification of the pion generators and the last term reflects the complexity of the condensates which is absent in basis (A). They are of the same order of $m$ and $\bar{\theta}$. Manipulations of these commutators yield, to $O(m ; \bar{\theta})$

$$
A\left(\eta \rightarrow \pi^{+} \pi^{-}\right)=\frac{-i}{F_{\pi}^{3}}\left\{\frac{i}{\sqrt{3}}\left(\beta_{u}-\beta_{d}\right)\left(-m_{u}\langle\bar{u} u\rangle+m_{d}\langle\bar{d} d\rangle\right)\right.
$$




$$
\left.-\frac{1}{\sqrt{3}}\left(m_{u}+m_{d}\right)\left(\left\langle\bar{u} \gamma_{5} u\right\rangle+\left\langle\bar{d} \gamma_{5} d\right\rangle\right)\right\}=\frac{K(m)}{\bar{m}} \bar{\theta} \frac{1}{F_{\pi}^{3}} \frac{2}{\sqrt{3}} C .
$$

In deriving the final step of (22) we have substituted in $\left\langle\bar{\psi}^{i} \psi^{i}\right\rangle=-C \cos \beta_{i} \simeq-C$ and $\left\langle\bar{\psi}^{i} i \gamma_{5} \psi^{i}\right\rangle=C \sin \beta_{i} \simeq C \beta_{i}$ and solution (12). We therefore confirm that CDVW's result is independent of chiral frames.

We conclude that the study of the vacuum orientation of the dynamical chiral symmetry breaking provides us an improved understanding of strong CP violation. It has been shown that $\eta \rightarrow 2 \pi$ decay occurs if $\bar{\theta}$ is non-zero. More precise experiment measuremental on the decay rate is encouraged to constrain $\bar{\theta}$.

Huang would like to thank F. Gilman for arranging him a visit to SSC Lab when this work was planned. A stimulation from S. Weinberg is gratefully acknowledged. 


\section{References}

[1] C. Vafa and E. Witten, Phys. Rev. Lett. 53 (1984) 535.

[2] The vacuum orientations when $\bar{\theta}=0$ have been discussed by P. Sikivie and C. B. Thorn, Phys. Lett. B234 (1990) 132.

[3] S. Adler, Phys. Rev. 137 (1969) 2426; J. Bell and R. Jackiw, Nuovo Cimento A60 (1969) 47; J. Wess and B. Zumino, Phys. Lett. B37 (1971) 95.

[4] Z. Huang, K. S. Viswanathan and D. D. Wu, Mod. Phys. Lett. A6 (1991) 711; Z. Huang and D.D. Wu, Commun. Theor. Phys. 12 (1991) 363.

[5] C. Callan, Jr., R. Dashen and D. Gross, Phys. Lett. B63 (1976) 334; R. Jackiw and C. Rebbi, Phys. Rev. Lett. 37 (1976) 72; S. Coleman, in Aspects of Symmetry ( Cambridge University Press, Cambridge, 1985).

[6] L. Brown, R. Carlitz, and C. Lee, Phys. Rev. D12 (1977) 413; R. Peccei and H. Quinn, Phys. Rev. Lett. 38 (1977) 1040; Phys. Rev. D12 (1977) 1391.

[7] M. Shifman, A. Vainshtein and V. Zakharov, Nucl. Phys. B126 (1980) 493.

[8] J. Nuyts, Phys. Rev. Lett. 26 (1971) 1204; V. Baluni, Phys. Rev. D19 (1979) 2227;

R. Crewther, in Field Theoretical Method in Particle Physics, NATO Advanced Study Institute, Kaiserslautern, Germany, ed. W. Ruhl ( Plenum Press, 1980); P. Di Vicchia and G. Veneziano, Nucl. Phys. B131 (1980) 253; E. Witten, Ann. Phys. (N. Y. ) 128 (1980) 363; G. 't Hooft, Phys. Rep. 102 (1986) 357.

[9] R. Dashen, Phys. Rev. D3 (1971) 1479.

[10] R. Crewther, P. Di Vicchia, G. Veneziano, and E. Witten, Phys. Lett. B88 (1979) 123.

[11] A. Abada, J. Galland, A. Le Younac, O. Oliver, O. Pene and J. Raynal, Phys. Lett. B256 (1991) 508; Orsay Preprint LPTHE 90/53 (1991); H. Banerjee, D. Chartterjee, and P. 
Mitra, Saha Institute Preprint, SINP-TNP-90/5; 90/13 (1991); S. Aoki, A. Gochsch, A. Manohar and S. Sharpe, Phys. Rev. Lett. 65 (1990) 1092. 